\title{
Transient eyelid retraction in myasthenia gravis
}

\author{
J. E. PUKLIN, J. G. SACKS ${ }^{1}$, AND B. BOSHES \\ From the Departments of Ophthalmology and Neurology, Northwestern Medical School, \\ Chicago, Illinois, U.S.A.
}

SYNOPSIS Three patients with myasthenia gravis had transient retraction of the upper eyelids which usually followed prolonged upgaze and which persisted for many seconds. This could result from post-tetanic facilitation, myotonia, or a transient contracture. Retraction of this type is to be $s$ distinguished from both the long-standing unilateral retraction associated with ptosis of the contra- $\overrightarrow{.}$ lateral eyelid and the fleeting lid twitch sign.

Retraction of the upper eyelids in myasthenia gravis is so uncommon and seemingly paradoxical that its presence might inappropriately make myasthenia gravis one of the least likely considerations in the differential diagnosis of a patient's neuromuscular disorder. This is especially true because myotonic lid lag occurs in the hyperkalaemic form of periodic paralysis (Layzer et al., 1967) and in its hypokalaemic type (Resnick and Engel, 1967). Although this unusual sign has been previously reported to occur in myasthenia gravis (Buzzard, 1900; Bramwell, 1927; Walsh, 1943; Chavanne et al., 1955; Cogan, 1965; Gay et al., 1967), other authors have not distinguished between fleeting, transient, and long-standing retraction. We believe that separate mechanisms underly each of these three categories. It is our purpose to call attention to the unusual association of upper eyelid retraction with myasthenia gravis and to comment on the mechanisms which possibly underlie this clinical sign.

\section{CASE 1}

(CWMH 2-023836-0) A 50 year old man had been well until September 1968 when he awoke with ptosis of the left upper eyelid. A diagnosis of myasthenia gravis was made elsewhere and the patient was treated with neostigmine bromide. After initial improvement, the patient's condition deteriorated necessitating a tracheostomy in December 1968.

\footnotetext{
1 Address for reprint requests: Dr J. G. Sacks, 303 East Chicago Avenue, Chicago, Illinois 60611, U.S.A.

(Accepted 15 August 1975.)
}

In January 1969, the patient was seen at the Mayo Clinic where a diagnosis of myasthenia gravis was made. Electromyographic tests performed there showed a definite decrement in the compound $\vec{f}$ muscle action potentials of the thenar and hyp\& thenar muscles during $2 \mathrm{~Hz}$ stimulation of ttge median and ulnar nerves respectively. Intracellular microelectrode studies of external intercostal muscte obtained at biopsy showed low amplitude miniatue endplate potentials.

He was found to have a thymoma which we removed. It weighed $40 \mathrm{~g}$.

Except for occasional diplopia and dysphagia the patient slowly improved.

When we first examined the patient in April 1971 he was receiving neostigmine and ephedrine. He had no diplopia, but abduction nystagmus was present $\mathbb{D}$ in the left eye; there was no accompanying delay of abduction of the right eye. During the examination we observed at various times a right ptosis, a left ptosis, a bilateral lid retraction, and an alternating asymmetrical lid retraction (Fig. 1).

It seemed as though prolonged upgaze made the retraction more likely to appear. Neither interrupting. fixation of either eye nor intravenous injection of $\exists$ edrophonium chloride had any effect on the retraction. At various times, edrophonium chloride $\operatorname{did} \bigcirc$ beneficially affect the ptosis and voice.

Other positive neurological findings included nasal speech, an inability to elevate the soft palate, and absence of the gag reflex. General muscle strength was normal but easy fatiguability could be demon- $N$ strated.

The blood count, urinalysis, electrocardiogram, N chest radiographs, serum glutamic oxaloacetic ${ }^{\mathrm{C}}$ transaminase, lactate dehydrogenase, serum potas 2 sium, and thyroxine were normal. 


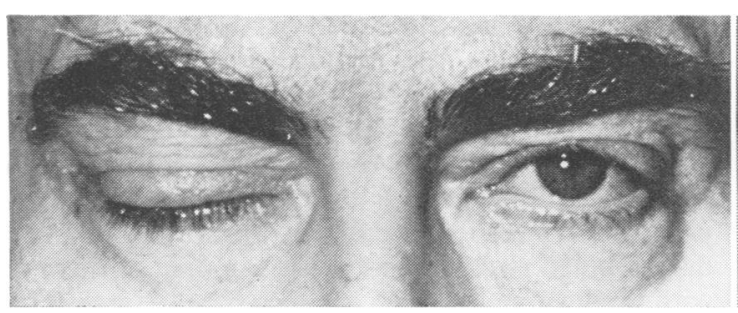

(a)

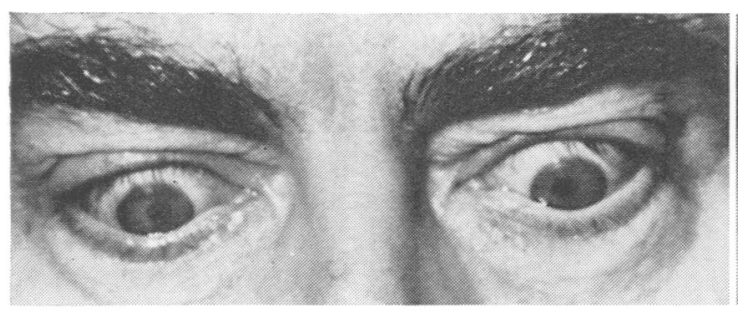

(c)

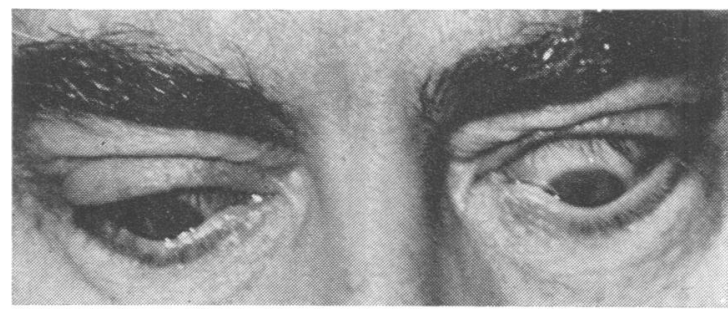

(e)

FIG. 1 Case 1. Eyelid positions.
(a) Right ptosis,
(b) Left ptosis.
(c) Bilateral eyelid retraction.
(d) and (e) Asymmetrical eyelid retraction.
(Reproduced from Kodachrome movie film).

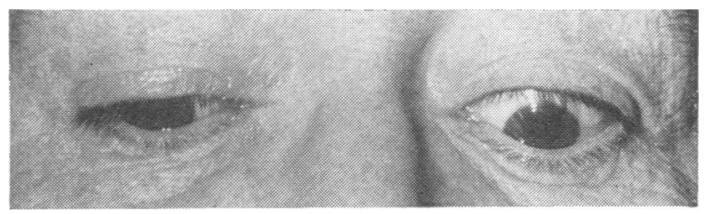

FIG. 2 Case 2. Left eyelid retraction.

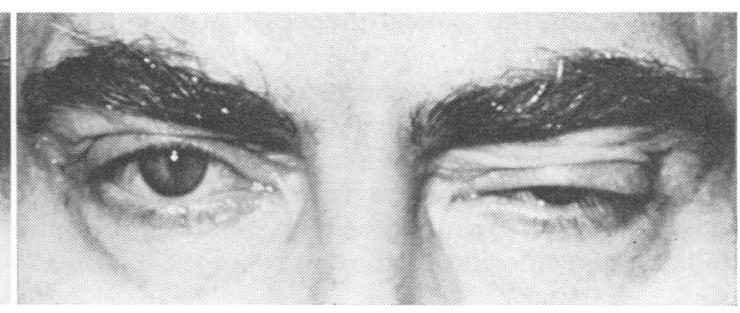

(b)

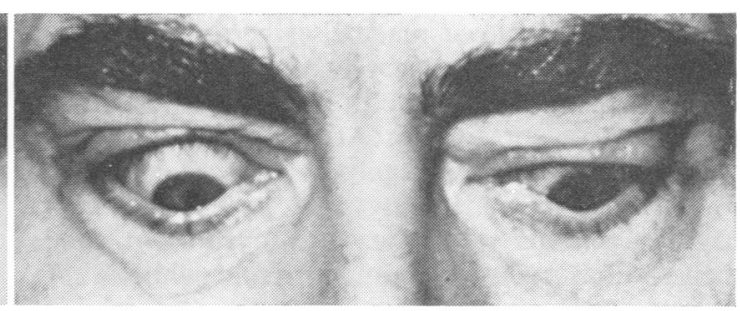

(d)

CASE 2

(CWMH 2041037-3) A 50 year old woman had been well until 1969 when she developed intermittent diplopia. In October 1971 her symptoms worsened and were noted to be more severe as the day progressed. She was therefore hospitalized for further evaluation. No medications for the weakness were being given at the time of examination.

Visual acuity was $6 / 6$ in each eye. The left upper eyelid was ptotic. The eyes were straight in the primary position. On upgaze a 12 to 15 prism dioptre left hypotropia was present; after prolonged upgaze the hypotropia increased. Then, as the patient looked down, retraction of the left upper eyelid occasionally occurred in both the primary position and in downgaze (Fig. 2). When the eyes returned to the primary position from downgaze, the upper eyelids briefly retracted and then came down (the 'lid twitch' sign of Cogan). Interrupting fixation and the intravenous administration of edrophonium chloride had no effect on these findings. The remainder of the physical examination was normal.

The patient had a mild iron deficiency anaemia. The urinalysis, electrocardiogram, chest radiograph, cerebrospinal fluid, serum potassium, and brain scan were normal. A left cerebral angiogram showed generalized atherosclerosis. An electroencephalogram showed nonspecific subcortical changes in the left hemisphere. A Jolly test showed a $20 \%$ decrement in the right abductor digiti minimi even though the patient was receiving pyridostigmine, thus help- 
ing to confirm the clinical diagnosis of myasthenia gravis.

The patient has been seen regularly at the Northwestern University Medical Clinics since her discharge from the hospital. While she has not developed evidence of myasthenia gravis anywhere else in her body, she has required either neostigmine or ambenonium chloride for the control of the diplopia.

\section{CASE 3}

(NMH 2-050333-4) A 50 year old woman developed diplopia at the age of 22 years and a diagnosis of myasthenia gravis was made elsewhere. She was not treated for 14 years; then neostigmine methylsulphate therapy was started. Later, pyridostigmine bromide was substituted. At the age of 45 years diabetes mellitus was diagnosed and was controlled by diet. A thyroidectomy was performed for a colloid adenoma when she was 48 years old. Since then she has been clinically euthyroid while taking sodium levothyroxine $0.3 \mathrm{mg}$ daily.

She experienced infrequent intermittent ptosis and the diplopia became more annoying. She was referred to Northwestern Memorial Hospital for evaluation and was receiving pyridostigmine bromide at the time of examination in March 1972.

The best corrected visual acuity was $5 / 6$ and $6 / 6$ in the right and left eyes respectively. Exophthalmometry readings were $16 \mathrm{~mm}$ in each eye, which is normal. There was slight but definite weakness of both lateral recti. A left hypertropia of 4 prism dioptres in the primary position and 6 prism dioptres on left gaze was noted. In the primary position she showed neither ptosis nor eyelid retraction. The bilateral lid twitch response of Cogan was present. After prolonged upgaze, bilateral eyelid retraction was present both in the primary position and in downgaze. Occasionally eyelid retraction was unilateral (Fig. 3); neither interrupting fixation nor intravenous injection of edrophonium chloride had any effect on the eyelid retraction, although after the injection the diplopia disappeared.

The blood count, urinalysis, serum potassium, and chest radiograph were normal. An electrocardio-

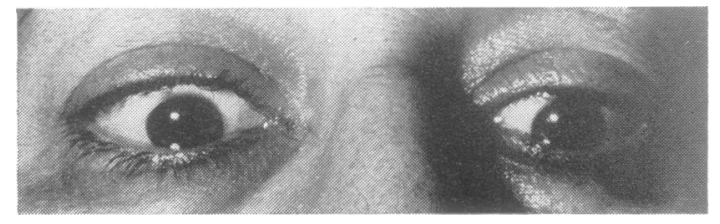

FIG. 3 Case 3. Unilateral eyelid retraction. gram revealed an old anterior wall infarction. A glucose tolerance test confirmed the presence of diabetes mellitus. A $15.8 \%$ decrement was noted on a Jolly test performed on the abductor digiti minimi.

The patient was readmitted to Northwestern Memorial Hospital in November 1973 with complaints of generalized weakness. Pyridostigmine was discontinued and she was given a course of therapy with prednisone to which she responded favourably.

\section{DISCUSSION}

Retraction of the upper eyelids is frequently due to a dysthyroid state. However, lid retraction of endocrine origin persists for months to years and should not be confused with the transient phenomenon we have observed in patients with myasthenia gravis, especially since our patients seemed to be euthyroid.

Three distinct types of eyelid retraction in myasthenia gravis can now be distinguished on the basis of the duration of the retraction transient, which occurs over many seconds fleeting, which lasts for less than one second and long-standing, which persists for months of years.

The first type, transient, is represented by ouf patients. To our knowledge, this type has no been reported previously in myasthenia gravis Transient eyelid retraction may be due to posttetanic facilitation of the levator palpebrae superioris, similar to the mechanism described in small muscles of the hands of myasthenics by Desmedt (1959), and in an in vitro preparation by Elmqvist et al. (1964). In our patients the retraction was most likely to appear after either prolonged upgaze or staring straight ahead. These activities cause prolonged contraction of the levator and may be similar to a machineinduced tetany.

Tending to point away from this hypothesis, however, is the fact that the retraction was present in downgaze. Since Loeffler et al.(1966) demonstrated that the levator in normal patients has little or no electrical activity in downgaze, it might be inferred that its motor nerve is electrically silent. By definition, the concept of in vivo post-tetanic facilitation requires that the muscle be stimulated by a neural impulse.

Myotonia or a transient contracture of the levator are alternative explanations. Myotonia is a delay in relaxation of contracted muscle, 
while contracture is a mechanical shortening of muscle which is maintained without accompanying muscle action potentials. The two could theoretically be distinguished electromyographically, but the levator palpebrae superioris does not lend itself to routine electrophysiological study. More importantly, our concern here is with the clinical significance of a readily observable sign.

The second type, fleeting, has been described by Cogan (1965) as the 'lid twitch'. This is essentially an overshoot of the upper eyelids when the eyes are directed to the primary position from downgaze. Since the levators are at rest in downgaze, they momentarily respond more vigorously than they otherwise would.

The third type, long-standing retraction, has been the most commonly reported variety in myasthenia gravis and is associated with weakness of the contralateral levator palpebrae superioris (Buzzard, 1900; Walsh, 1943; Chavanne et al., 1955; Gay et al., 1967). The explanation offered by Gay et al. (1967) seems most acceptable in these cases. They hypothesized that the levator muscles are true yoke muscles which follow Hering's law of equal innervation. They believed that the myasthenic defect in the ptotic lid should lead to increased innervation of both levators, resulting in retraction of the stronger eyelid. In confirmation of this, they noted that covering the eye which had the ptotic lid lessened the contralateral retraction. When edrophonium chloride was administered, the ptosis improved and the contralateral retraction was relieved.

To help validate the distinction between cases of transient and long-standing lid retraction, we administered edrophonium chloride intravenously to our patients. This had no effect on the retraction, thus tending to support our contention.

Finally, transient retraction with generalized muscular weakness should no longer be considered to be a sign peculiar to periodic paralysis. Layzer et al. (1967) considered that the lid lag was possibly unique to the hyperkalaemic form of this disease. Later, however, Resnick and Engel (1967) showed it to be present in the hypokalaemic form, thus indicating that the sign is not a reliable indicator of the type of periodic paralysis. They attributed the retraction to myotonia, but did not test the hypothesis electromyographically, presumably because of the difficulties inherent in such studies of the levator.

\section{REFERENCES}

Bramwell, E. (in discussion of Collier, J.) (1927). Nuclear ophthalmoplegia with especial reference to retraction of the lids and ptosis and to lesions of the posterior commissure. Brain, 50, 488-498.

Buzzard, T. (1900). Clinical lecture on cases of myasthenia gravis pseudo-paralytica. British Medical Journal, 1, 493496.

Chavanne, H., Girard, P. F., and Rougier, J. (1955). Rétraction palpébrale et myasthénie oculaire. Revue d'Oto-Neuroophthalmologie, 27, 18-21.

Cogan, D. G. (1965). Myasthenia gravis: A review of the disease and a description of lid twitch as a characteristic sign. Archives of Ophthalmology, 74, 217-221.

Desmedt, J. E. (1959). The physio-pathology of neuromuscular transmission and the trophic influence of motor innervation. American Journal of Physical Medicine, 38, 248-261.

Elmqvist, D., Hofmann, W. W., Kugelberg, J., and Quastel, D. M. J. (1964). An electrophysiological investigation of neuromuscular transmission in myasthenia gravis. Journal of Physiology, 174, 417-434.

Gay, A. J., Salmon, M. L., and Windsor, C. E. (1967). Hering's law, the levators, and their relationship in disease states. Archives of Ophthalmology, 77, 157-160.

Layzer, R. B., Lovelace, R. E., and Rowland, L. P. (1967). Hyperkalemic periodic paralysis. Archives of Neurology, 16, 455-472.

Loeffler, J. D., Slatt, B., and Hoyt, W. F. (1966). Motor abnormalities of the eyelids in Parkinson's disease. Archives of Ophthalmology, 76, 178-185.

Resnick, J. S., and Engel, W. K. (1967). Myotonic lid lag in hypokalaemic periodic paralysis. Journal of Neurology, Neurosurgery, and Psychiatry, 30, 47-51.

Walsh, F. B. (1943). Myasthenia gravis and its ocular signs: A review. Transactions of the American Ophthalmological Society, 41, 556-624. 